\title{
Catastrophic stroke burden in a patient with uncontrolled psoriasis and psoriatic arthritis: a case report
}

Joline M. Fan ${ }^{*}$, David A. Solomon², Giselle Y. López³, Jeffrey W. Hofmann², Rene A. Colorado ${ }^{4}$, Anthony S. Kim', Karl Meisel ${ }^{1}$ and Cathra Halabi ${ }^{1,5}$

\begin{abstract}
Background: Psoriasis is the most common chronic inflammatory condition involving the Thelper cell system. Population studies have demonstrated that patients with psoriasis and/or psoriatic arthritis have an increased risk of developing vascular risk factors, including diabetes, hypertension, and obesity, and increased risk of adverse vascular events, including myocardial infarction and stroke. Population studies have generally investigated the individual contributions of psoriasis and psoriatic arthritis to development of vascular risk factors; fewer studies have investigated the additive contribution of comorbid inflammatory disorders. We present a case of a woman with psoriasis, psoriatic arthritis, and comorbid vascular risk factors.

Case presentation: A 49 year-old Caucasian woman with a history of severe psoriasis and psoriatic arthritis since adolescence presented with bilateral lower extremity weakness. She was found to have acute bilateral watershed infarcts and multifocal subacute infarcts. Her evaluation revealed vascular risk factors and elevated non-specific systemic inflammatory markers; serum and cerebral spinal fluid did not reveal underlying infection, hypercoagulable state, or vasculitis. Over the course of days, she exhibited precipitous clinical deterioration related to multiple large vessel occlusions, including the bilateral anterior cerebral arteries and the left middle cerebral artery. Autopsy revealed acute thrombi and diffuse, severe atherosclerosis.
\end{abstract}

Conclusion: Patients with early onset inflammatory disease activity or comorbid inflammatory disorders may have an even higher risk of developing metabolic syndrome and adverse vascular events compared to patients with lateonset disease activity or with a single inflammatory condition. The described case illustrates the complex relationship between inflammatory disorders and vascular risk factors. The degree of systemic inflammation, as measured by severity of disease activity, has been shown to have a dose-response relationship with comorbid vascular risk factors and vascular events. Dysregulation of the Th1 and Th17 system has been implicated in the development of atherosclerosis and may explain the severe atherosclerosis seen in such chronic inflammatory conditions. Further research will help refine screening and management guidelines to account for comorbid inflammatory disorders and related disease severity.

Keywords: Atherosclerosis, Stroke, Psoriasis, Psoriatic arthritis

\footnotetext{
* Correspondence: joline.fan@ucsf.edu

'Department of Neurology, University of California, San Francisco, California,

USA

Full list of author information is available at the end of the article
}

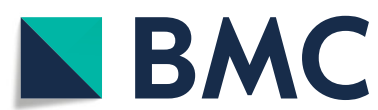

(c) The Author(s). 2020 Open Access This article is licensed under a Creative Commons Attribution 4.0 International License, which permits use, sharing, adaptation, distribution and reproduction in any medium or format, as long as you give appropriate credit to the original author(s) and the source, provide a link to the Creative Commons licence, and indicate if changes were made. The images or other third party material in this article are included in the article's Creative Commons licence, unless indicated otherwise in a credit line to the material. If material is not included in the article's Creative Commons licence and your intended use is not permitted by statutory regulation or exceeds the permitted use, you will need to obtain permission directly from the copyright holder. To view a copy of this licence, visit http://creativecommons.org/licenses/by/4.0/ The Creative Commons Public Domain Dedication waiver (http://creativecommons.org/publicdomain/zero/1.0/) applies to the data made available in this article, unless otherwise stated in a credit line to the data. 


\section{Background}

Psoriasis is a common chronic inflammatory disorder affecting approximately $1.5-3 \%$ of the adult population [1, 2]. An additional $6-30 \%$ of patients with psoriasis also have psoriatic arthritis, which may reflect a more pronounced systemic disease [3-5]. Population cohort studies have identified both psoriasis and psoriatic arthritis to be individual risk factors for vascular disease [4-7]; however, the contribution of comorbid inflammatory diseases for clinical screening and management guidelines remains unknown. Here, we present an illustrative report of a fatal stroke in a young patient with severe psoriasis, psoriatic arthritis, and metabolic syndrome.

\section{Case presentation}

A 49 year-old Caucasian woman with psoriasis, psoriatic arthritis, multivessel coronary artery disease, hypertension, subclinical hypothyroidism, and diabetes mellitus presented with bilateral lower extremity weakness and severe anemia. Regarding her history of psoriasis, she initially developed diffuse psoriatic plaques and axial psoriatic arthritis at age 19. Her first severe psoriasis flare occurred at age 29. Chart review did not reveal recorded Psoriasis Area and Severity Index (PASI) scores but there was documentation of erythema and pustular psoriasis measured over $70 \%$ of her body surface area with elevated white blood cell count. Despite treatment with prednisone and acitretin, after 1 year she developed severe cutaneous flares of pustular psoriasis measuring up to $90 \%$ of total body surface area with spared regions in her legs, necessitating multiple hospitalizations. Her treatment was escalated to Geockerman therapy, methotrexate, and topical steroids, in addition to prednisone and acitretin. In subsequent years, her chronic psoriatic skin manifestations involved roughly $30 \%$ of her total body surface area, meeting criteria for severe psoriasis, and these skin manifestations were managed primarily with topical steroids. Plain films ultimately revealed active inflammatory spondylitis. Despite recommendations to start disease modifying therapy, the patient declined further treatment.

Six years prior to the most recent presentation, the patient was diagnosed with metabolic syndrome. Risk factors measured at the time of diagnosis included a peak hemoglobin A1c level of $11.6 \%$, body mass index of 36 , triglycerides of 310 , high density lipoprotein (HDL) of 34 , and systolic blood pressures routinely measured between 140 and 160. For her modifiable risk factors of diabetes, dyslipidemia, and hypertension, she was prescribed insulin, statins, and antihypertensive agents, respectively. Her family history was notable for ischemic stroke in her mother though of unknown etiology and age. There were no known inflammatory disorders in the family. Three months prior to the most recent presentation, the patient was hospitalized for a non-ST elevation myocardial infarction (NSTEMI). Cardiac catheterization revealed severe right coronary artery (RCA) disease and in-stent thrombosis of a pre-existing stent within the left circumflex artery placed 6 years prior. She underwent re-stenting for her left circumflex artery and RCA and was subsequently treated with a dual antiplatelet therapy regimen with aspirin and clopidogrel.

Subsequently, she presented with subacute bilateral lower extremity weakness and confusion. Her brain MRI revealed acute bilateral watershed infarcts, in addition to subacute left parietal and frontal gyrus infarcts. CT angiography of the head and neck revealed diffuse atherosclerotic plaques in the aortic arch and carotid bulbs, occlusion of the left internal carotid artery (ICA), and narrowing of the bilateral M1 segments of the middle cerebral artery (MCA). (Fig. 1a-c). There was reconstitution of flow in the left cavernous and supraclinoid segments of the ICA via an intact Circle of Willis. In addition to the bilateral MCA narrowing, there was mild narrowing of the right carotid artery and a single

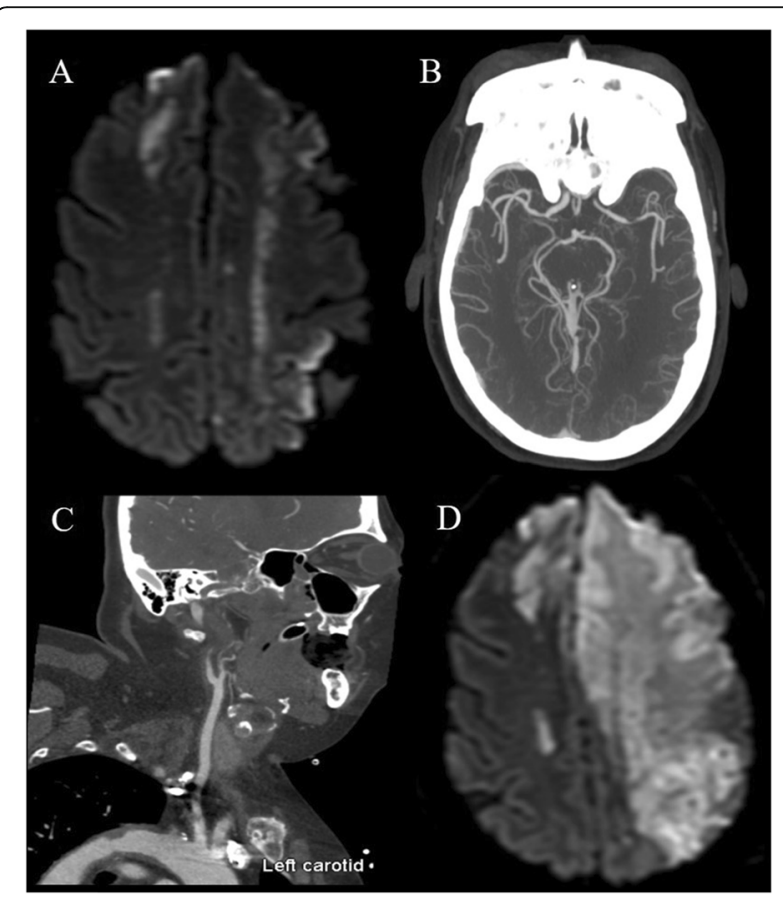

Fig. 1 a Diffusion-weighted axial MRI showing bilateral anterior cerebral artery (ACA) and middle cerebral artery (MCA) distribution infarcts. $\mathbf{b}$ CTA axial MIP demonstrating mild irregular luminal narrowing of the right $\mathrm{M} 1$ segment and severe focal narrowing of the distal left M1 segment. C CTA sagittal MIP demonstrating calcified atherosclerotic plaque of the left carotid bulb and complete occlusion of the proximal cervical left internal carotid artery. $\mathbf{d}$ Diffusion-weighted axial MRI showing marked interval progression of infarcts involving the bilateral ACA and left MCA distributions 
diminutive A1 segment which perfused bilateral anterior cerebral arteries (ACA) vessels.

There were no known preceding hemodynamic changes or systemic illnesses. Possible etiologies of her multifocal infarcts included severe atherosclerosis and related artery-artery thromboembolic events, cardioembolic events, hypercoagulable state, infection, or vasculitis. Cardiac rhythm telemetry did not reveal atrial fibrillation. Transthoracic echocardiogram revealed normal left ventricular function, normal left and right atrial size, no thrombus, and no intra- or extra-cardiac shunt. Subsequent transesophageal echocardiogram (TEE) again did not reveal thrombus or valvular lesions. The TEE also demonstrated atheromatous plaque in the proximal descending aorta greater than $4 \mathrm{~mm}$ in thickness. Laboratory studies included hemoglobin $(\mathrm{Hg})$ of $4.2 \mathrm{~g} / \mathrm{dL}$, an elevated erythrocyte sedimentation rate > $100 \mathrm{~mm} / \mathrm{hr}$. (normal $0-15 \mathrm{~mm} / \mathrm{hr}$ ) and lipoprotein (a) $133 \mathrm{nmol} / \mathrm{L}$ (normal $<75 \mathrm{nmol} / \mathrm{L}$ ) but were otherwise unrevealing (Table 1). The cerebral spinal fluid (CSF) did not have infectious or inflammatory properties. Workup of anemia was consistent with alpha thalassemia and severe iron deficiency, also supported by peripheral blood smear.

Despite aggressive medical management, the patient clinically deteriorated. Repeat imaging revealed marked interval infarct progression involving multiple vascular territories (Fig. 1d). She underwent a left-sided decompressive hemicraniectomy as a life sustaining maneuver, and concomitant brain biopsy did not demonstrate evidence of vasculitis or infection. Clinical course was further complicated by sepsis, renal failure, and an acute generalized exanthematous pustulosis drug reaction to iodine contrast. In accordance with the patient's prior wishes, she was transitioned to comfort-focused care and her family agreed to an autopsy.

The autopsy revealed eccentric, firm, yellow plaques involving all proximal intracranial vessels, consistent with severe atherosclerosis. There was an occlusive thrombus of the right ACA as well as diffuse arteriolar microthrombi. Elements of acute and chronic ischemic changes were seen on histology, including extensive ischemic neuronal necrosis. Diffuse, severe atherosclerosis was determined to be the cause of fatal burden of ischemic stroke (Fig. 2).

\section{Discussion and conclusions}

We present a case of a young patient with psoriasis and psoriatic arthritis, severe and diffuse atherosclerosis, and ultimately a fatal burden of ischemic infarcts of the brain. While the definitive contributions of the patient's individual inflammatory conditions and anemia are unknown, we suggest that severe psoriasis and psoriatic arthritis enhance and accelerate progression of early presence of vascular risk factors and that recognition of this relationship provides another opportunity for risk factor stratification and treatment. Psoriasis and psoriatic arthritis have each been associated with cardiovascular risk factors and metabolic syndrome. This report illustrates that the burden and severity of comorbid inflammatory conditions and metabolic syndrome should prompt aggressive screening and management of vascular risk factors.

\section{Pathophysiology}

The pathophysiology of psoriasis involves the activation of effector $\mathrm{T}$ lymphocytes, including $\mathrm{T}$ helper cell type 1 and 17. Skin infiltration of activated $\mathrm{T}$ cells promotes antigen presentation and propagation of inflammatory cytokines [3]. A dysregulated Th1 system within the vessel wall intimal layer is implicated in the development of atherosclerosis and is a biologically plausible explanation for accelerated atherosclerosis in a chronic inflammatory state $[8,9]$. The initial lesion responsible for atherogenesis is the fatty-streak, which is pathologically composed of monocytes and T lymphocytes $[8,10]$. Endothelial cell activation leads to an enhanced local inflammatory response with upregulation of leukocyte adhesion molecules, increased of leukocyte recruitment, and expansion of the subendothelial fatty streak, leading to plaque formation $[10,11]$. Progressive focal narrowing of vessels is punctuated by exposure of thrombogenic material when there is intimal layer plaque rupture or endothelial cell layer disruption. Thrombosis then leads to conditions such as unstable angina, myocardial infarction, or stroke $[11,12]$. Fig. 2 illustrates extensive atheromas (2a) and chronic changes to the vessel wall layers (2c-d). Figure 2e illustrates the presence of $\mathrm{T}$ lymphocytes and macrophages within the thickened intima, which can be seen within the natural process of atherosclerosis [8, 10]. There is no evidence of excessive or patterned presence of inflammatory cells in pathology specimens, suggesting a chronic, cumulative process, and highlighting indirect and potentially synergistic roles of systemic inflammation in accelerating atherosclerosis.

Circulation of inflammatory substances including low density lipoprotein (LDL) and lipoprotein (a) have also been directly implicated in vessel wall reactivity and plaque formation [3]. Studies have shown increased subclinical atherosclerosis and increased intima-medial thickness in patients with psoriatic arthritis, but without otherwise clear cardiovascular risk factors [13, 14], illustrating a more direct effect of systemic inflammation on the arterial wall. More severe subclinical atherosclerosis has also been seen in patients with psoriatic arthritis as compared to control populations and patients with cutaneous psoriasis alone [12]. The severity of atherosclerosis may increase with the added burden of inflammatory joint disease in patients with psoriasis. 
Table 1 Summary of serum and CSF studies obtained on present hospitalization

\begin{tabular}{|c|c|c|}
\hline & Data & Reference \\
\hline \multicolumn{3}{|l|}{ Lipid profile } \\
\hline Cholesterol, Total & 179 & $<200 \mathrm{mg} / \mathrm{dL}$ \\
\hline Triglycerides & 253 & $<200 \mathrm{mg} / \mathrm{dL}$ \\
\hline LDL & 114 & $<130 \mathrm{mg} / \mathrm{dL}$ \\
\hline Lipoprotein a & 133 & $<75 \mathrm{nmol} / \mathrm{L}$ \\
\hline \multicolumn{3}{|l|}{ Hypercoagulation } \\
\hline Beta-2-glycoprotein Antibody, lgG & $<10.0$ & $<20.1 \mathrm{CU}$ \\
\hline Beta-2-glycoprotein Antibody, IgM & $<5.0$ & $<20.1 \mathrm{CU}$ \\
\hline Protein C, Activity & 99 & $76-146 \%$ \\
\hline Anti-Cardiolipin Antibody, IgG & 15.8 & $<20.1 \mathrm{CU}$ \\
\hline Anti-Cardiolipin Antibody, IgM & $<10.0$ & $<20.1 \mathrm{CU}$ \\
\hline Lupus Anticoagulant by HEXA & Neg & Neg \\
\hline RWT Seconds & 30.9 & $29.0-44.0 \mathrm{~s}$ \\
\hline \multicolumn{3}{|l|}{ CSF studies } \\
\hline WBC & 1 & $<6 \times 10 \mathrm{E} 6 / \mathrm{L}$ \\
\hline $\mathrm{RBC}$ & 44 & $0 \times 10 \mathrm{E} 6 / \mathrm{L}$ \\
\hline Glucose & 76 & $40-70 \mathrm{mg} / \mathrm{dL}$ \\
\hline Protein & 13 & $15-50 \mathrm{mg} / \mathrm{dL}$ \\
\hline IgG Index & 0.6 & 0.3-0.6 Ratio \\
\hline Serum glucose & 164 & $<200$ \\
\hline Rapid HSV-1 PCR & Neg & Neg \\
\hline Rapid HSV-2 PCR & Neg & Neg \\
\hline VZV PCR & Neg & Neg \\
\hline VZV IgM & 0.03 & $<0.9$ \\
\hline \multicolumn{3}{|l|}{ Autoimmune/Endocrine } \\
\hline Hemoglobin A1c & 6.4 & $4.3-5.6 \%$ \\
\hline TSH & 0.64 & $0.45-4.12 \mathrm{mlU} / \mathrm{L}$ \\
\hline Free T4 & 13 & 10-18 pmol/L \\
\hline CRP & $5.4->36.9$ & $<6.3$ \\
\hline ESR & $>100$ & $0-15 \mathrm{~mm} / \mathrm{h}$ \\
\hline Anti-Nuclear Antibodies & $<40$ & $<40$ \\
\hline Anti Proteinase $3 \mathrm{Ab}$ & $<10.0$ & $<20.0 \mathrm{CU}$ \\
\hline Anti Myeloperoxidase & $<10.0$ & $<20.0 \mathrm{CU}$ \\
\hline GAD-65 Autoantibodies & $<1.0$ & $<1.0 \mathrm{U} / \mathrm{mL}$ \\
\hline Insulin Autoantibody & $<0.4$ & $<0.4 \mathrm{U} / \mathrm{mL}$ \\
\hline Islet Cell Antigen 512 Antibody & $<0.8$ & $<0.8 \mathrm{U} / \mathrm{mL}$ \\
\hline Rheumatoid Factor, serum & $<40$ & $<40 \mathrm{IU} / \mathrm{mL}$ \\
\hline Complement C3, serum & 101 & $71-159 \mathrm{mg} / \mathrm{dL}$ \\
\hline Complement C4, serum & 26.4 & $13-30 \mathrm{mg} / \mathrm{dL}$ \\
\hline Cryoglobulin & $<0.12$ & $<0.12 \mathrm{~g} / \mathrm{L}$ \\
\hline Heterophile Agglutination & Neg & Neg \\
\hline
\end{tabular}

Table 1 Summary of serum and CSF studies obtained on present hospitalization (Continued)

\begin{tabular}{lll}
\hline & Data & Reference \\
\hline Infectious & & \\
Hepatitis B sAb & $<5$ & $\mathrm{mlU} / \mathrm{mL}$ \\
Hepatitis B CAb & Neg & Neg \\
Hepatitis B sAg & Neg & Neg \\
Hepatitis C Antibody & Neg & Neg \\
HIV Ag/Ab & Neg & Neg \\
\hline
\end{tabular}

\section{Psoriasis}

Population studies have demonstrated that patients with psoriasis have increased cardiovascular risk factors, including diabetes, hypertension, hyperlipidemia, and obesity, which are all features of metabolic syndrome [15-18]. Across observational studies, meta-analyses describe a 1.4-2.2 fold increased risk of developing metabolic syndrome in patients with psoriasis as compared to controls [18, 19]. In addition, the risk of developing metabolic syndrome has been shown to increase with psoriasis severity, with odds ratios (OR) of 1.22, 1.56, 1.98 for mild, moderate, and severe psoriasis, respectively [18]. Other studies have noted specific risk factors that are more prevalent in severe versus mild psoriasis, including diabetes (OR 1.39, 95\% CI 1.22-1.58) and obesity (OR 1.47, 95\% CI 1.32-1.63) [20]. These studies illustrate the relationship between the degree of systemic inflammation and development of metabolic syndrome.

In addition to the added risk conferred from elevated cardiovascular risk factors, population studies have also demonstrated that psoriasis independently leads to an increased risk of myocardial infarction or stroke [7, 18, $21,22]$. After adjusting for cardiovascular risk factors, studies demonstrate that mild and severe psoriasis confer a 1.29-fold risk (95\% CI 1.02-1.63) and a 1.7-fold risk (CI 1.32-2.18) of myocardial infarction, as well as a 1.12-fold risk (95\% CI, 1.08-1.16) and a 1.56-fold risk (95\% CI 1.32-1.84) of stroke [23], respectively. These findings suggest a shared inflammatory pathway involved in the pathogenesis of psoriasis and atherogenesis [24].

The risk of cardiovascular risk factors and adverse events has also been noted to correlate with age of onset $[22,25]$. The risk of incident myocardial infarction (MI) was found to be higher in younger patients with severe psoriasis as compared to older patients with late-onset psoriasis (HR 3.10 in a 30 year-old vs 1.36 in a 60 yearold) [25]. The difference in the disease activity of psoriasis is dependent on both genetic and environmental factors. Detection of specific human leukocyte antigens (HLA), such as type Cw6, has been differentially found in patients with early onset psoriasis as compared to late 


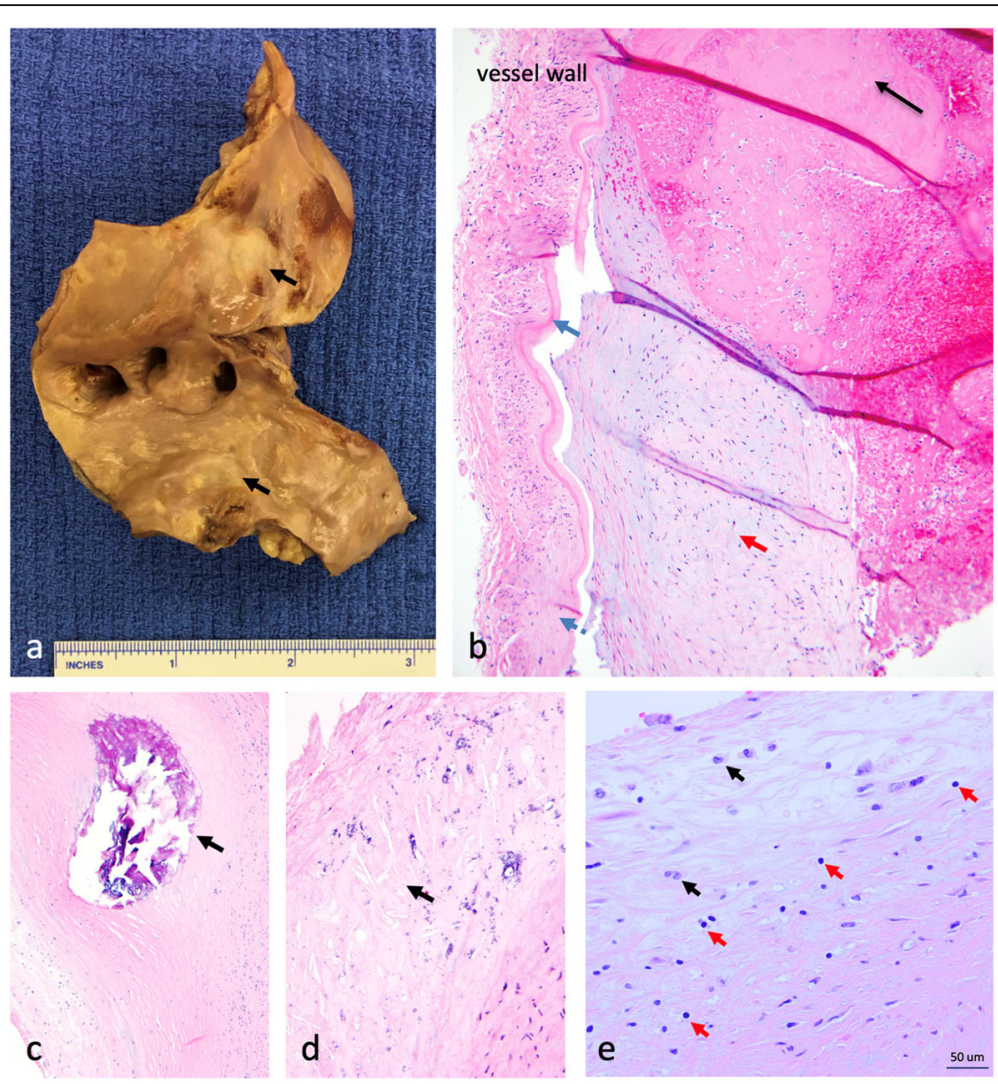

Fig. 2 Gross and microscopic pathology. a Gross pathology of aortic arch revealing extensive atheromas (black arrows). b Histopathology of the anterior cerebral artery. There is intimal thickening underlying the internal elastic lamina (blue arrow); the media and adventitia are distorted by fibrosis (blue dashed arrow). A recently-formed fibrin thrombus (black arrow) is adherent to a chronic and organized atherosclerotic plaque (red arrow). c Cross-sectional view of the left anterior descending coronary artery containing an intramural calcified nodule (black arrow). d Crosssectional view of the basilar artery wall, demonstrating architectural distortion with cholesterol clefts (black arrow) and microcalcifications. e Left anterior descending artery with thickening of the intima and inflammatory infiltrate including T lymphocytes (red arrows) and macrophages (black arrows)

onset psoriasis, as one example of biological differences that contribute towards disease activity [26].

\section{Psoriatic arthritis}

Other studies have looked at the contribution of psoriatic arthritis as an independent risk factor for metabolic syndrome and adverse cardiovascular events. Patients with psoriatic arthritis have increased prevalence of hypertension (OR 1.31, 95\% CI 1.26-1.47), hyperlipidemia (OR 1.23, 95\% CI 1.18-1.29), diabetes (OR, 1.38, 95\% CI 1.311.45), and obesity (OR 1.69, 95\% CI 1.62-1.75) [4]. The prevalence of MI (OR 1.36-fold, 95\% 1.04-1.77) and stroke (OR 1.26-fold, 95\% CI 1.03-1.71) was also higher in patients with psoriatic arthritis as compared to controls $[7,27]$. Notably, diagnosis of psoriatic arthritis did not confer a statistically significant elevation in mortality, as compared to controls [5]. Fewer studies have looked at the additive risk of accruing vascular risk factors in patients that have psoriatic arthritis in addition to cutaneous psoriasis. One study reports the elevated risk of vascular risk factors for patients with psoriatic arthritis to be 2.59fold (95\% CI 1.43-4.67) for cardiovascular disease, 2.42fold (95\% CI, 1.82-3.22) for hypertension, 1.9-fold (95\% CI, 1.22-2.96) for diabetes, and 1.58-fold (95\% CI, 1.19$2.09)$ for obesity $[28,29]$. The presence of multiple inflammatory conditions may imply presence of multiple vascular risk factors.

There are many contributing factors toward atherosclerosis and vascular events, including conventional risk factors of dyslipidemia and hypertension, family history, ethnicity, and lifestyle [30]. The potential impact of anemia [31] and hemoglobinopathy [32] is also acknowledged but not definitive. In this report, however, we have focused on addressing the direct and indirect contributions of psoriasis and psoriatic arthritis.

\section{Immunosuppressive therapy}

Methotrexate and tumor necrosis factor inhibitors lead to 0.72-fold (95\% CI 0.57-0.91) and 0.7-fold (95\% CI 0.54$0.9)$ respective reduction of incident vascular events 
including MI and stroke in rheumatic diseases while agents such as cyclosporine, oral retinoids, steroids, and non-steroidal anti-inflammatory agents may confer increased risk [33, 34]. While fewer studies have been performed in patients with psoriasis and psoriatic arthritis, data suggests that disease modifying therapies, biologics, and anti-inflammatory agents may also lead to a reduced rate of cerebrovascular risk factors and events [35-39].

In conclusion, psoriasis and psoriatic arthritis have both indirect and direct causative roles in increasing the risk of vascular disease. Younger patients or those with multisystem disease need aggressive screening and treatment for active inflammatory disease along with vascular risk factor stratification. In patients with inflammatory conditions, we suggest screening for modifiable vascular risk factors and treating the risk factors in accordance with contemporary guidelines. Similarly, in young patients with vascular risk factors, we suggest reviewing systems for undiagnosed inflammatory disease in the appropriate clinical context. Studies are needed to help identify appropriate immunosuppressive therapy selection for synergistic risk factor reduction. Studies are also needed to help identify timing, cost-effectiveness, and patient selection for screening and treatment strategies.

\section{Abbreviations}

ACA: Anterior cerebral artery; Cl: Confidence interval; CTA: Computerized tomography angiogram; HDL: High-density lipoprotein; Hg: Hemoglobin; HLA: Human leukocyte antigens; HR: Hazard ratio; ICA: Internal carotid artery; LDL: Low density lipoprotein; MCA: Middle cerebral artery; MI: Myocardial infarction; NSTEMI: Non-ST elevation myocardial infarction; OR: Odds ratios; PASI: Psoriasis Area and Severity Index; RCA: Right coronary artery;

TEE: Transesophageal echocardiogram

\section{Acknowledgements}

None.

\section{Authors' contributions}

JF, CH performed the data acquisition, analysis, and drafting of the manuscript. DS, GL, JH assisted with the analysis and interpretation of the pathology-specific data and the review of the manuscript. RC assisted with the data acquisition of the radiology images, figure creation, and the review of the manuscript. AK, KM, and $\mathrm{CH}$ assisted with the overall interpretation of the data and the review of the manuscript. All authors reviewed and approved of this manuscript.

\section{Funding}

None.

\section{Availability of data and materials}

All data generated or analyzed during this study are included in this published article.

\section{Ethics approval and consent to participate}

Not applicable; see consent for publication.

\section{Consent for publication}

Written informed consent was obtained from the next of kin for the publication of this case report, patient images, and lab data.

\section{Competing interests}

The authors declare that they have no competing interests.

\section{Author details}

'Department of Neurology, University of California, San Francisco, California, USA. ${ }^{2}$ Division of Neuropathology, Department of Pathology, University of California, San Francisco, California, USA. ${ }^{3}$ Division of Neuropathology, Department of Pathology, Duke University, Durham, North Carolina, USA. ${ }^{4}$ Department of Neurology, Salinas Valley Memorial Healthcare System, Salinas, California, USA. ${ }^{5}$ Weill Institute for Neurosciences, University of California, San Francisco, California, USA.

Received: 2 September 2019 Accepted: 10 March 2020

Published online: 21 March 2020

\section{References}

1. Gelfand JM, et al. Prevalence and treatment of psoriasis in the United Kingdom. Arch Dermatol. 2005;141:1537.

2. Kurd SK, Gelfand JM. The prevalence of previously diagnosed and undiagnosed psoriasis in US adults: results from NHANES 2003-2004. J Am Acad Dermatol. 2009;60:218-24.

3. Eder L, Gladman DD. Atherosclerosis in psoriatic disease: latest evidence and clinical implications. Ther Adv Musculoskelet Dis. 2015;7:187-95.

4. Jafri $\mathrm{K}$, et al. The incidence and Management of Cardiovascular Risk Factors in psoriatic arthritis and rheumatoid arthritis: a population-based study HHS public access. Arthritis Care Res. 2017;69:51-7.

5. Ogdie A, et al. Risk of major cardiovascular events in patients with psoriatic arthritis, psoriasis and rheumatoid arthritis: a population-based cohort study. Ann Rheum Dis. 2015;74:326-32.

6. Gladman DD, et al. Cardiovascular morbidity in psoriatic arthritis. Ann Rheum Dis. 2009;68:1131-5.

7. Gelfand JM, et al. The risk of stroke in patients with psoriasis. J Invest Dermatol. 2009;129(10):2411. https://doi.org/10.1038/jid.2009.112.

8. Stary HC, et al. A definition of initial, fatty streak, and intermediate lesions of atherosclerosis. A report from the committee on vascular lesions of the council on arteriosclerosis, American Heart Association. Circulation. 1994;89: 2462-78.

9. Matsuura $\mathrm{E}$, et al. Is atherosclerosis an autoimmune disease? BMC Med. 2014;12:47.

10. Ross R. Atherosclerosis - an inflammatory disease. N Engl J Med. 1999;340: $115-26$.

11. Hansson GK. Inflammation, atherosclerosis, and coronary artery disease. N Engl J Med. 2005;352:1685-95.

12. Eder $L$, et al. The burden of carotid artery plaques is higher in patients with psoriatic arthritis compared with those with psoriasis alone. Ann Rheum Dis. 2013;72:715-20.

13. Gonzalez-juanatey C, et al. High prevalence of subclinical atherosclerosis in psoriatic arthritis patients without clinically evident cardiovascular disease or classic atherosclerosis risk factors. Arthritis Rheum. 2007;57:1074-80.

14. Gonzalez-Juanatey C, et al. Endothelial dysfunction in psoriatic arthritis patients without clinically evident cardiovascular disease or classic atherosclerosis risk factors. Arthritis Rheum. 2007;57:287-93.

15. Love TJ, Qureshi AA, Karlson EW, Gelfand JM, Choi HK. Prevalence of the metabolic syndrome in psoriasis. Arch Dermatol. 2011;147:419.

16. Gisondi $\mathrm{P}$, et al. Prevalence of metabolic syndrome in patients with psoriasis: a hospital-based case? Control study. Br J Dermatol. 2007;157:68-73.

17. Wang $\mathrm{Y}$, et al. Psoriasis is associated with increased levels of serum leptin. $\mathrm{Br}$ J Dermatol. 2008;158:1134-5.

18. Armstrong AW, Harskamp CT, Armstrong EJ. Psoriasis and metabolic syndrome: a systematic review and meta-analysis of observational studies. J Am Acad Dermatol. 2013;68:654-62.

19. Rodríguez-Zúñiga MJM, García-Perdomo HA. Systematic review and metaanalysis of the association between psoriasis and metabolic syndrome. J Am Acad Dermatol. 2017;77:657-666.e8.

20. Neimann AL, et al. Prevalence of cardiovascular risk factors in patients with psoriasis. J Am Acad Dermatol. 2006;55:829-35.

21. Xu T, Zhang Y-H. Association of psoriasis with stroke and myocardial infarction: meta-analysis of cohort studies. Br J Dermatol. 2012;167:1345-50.

22. Horreau C, et al. Cardiovascular morbidity and mortality in psoriasis and psoriatic arthritis: a systematic literature review. J Eur Acad Dermatology Venereol. 2013;27:12-29.

23. Armstrong EJ, Harskamp CT, Armstrong AW. Psoriasis and major adverse cardiovascular events: a systematic review and meta-analysis of observational studies. J Am Heart Assoc. 2013;2:e000062. 
24. Armstrong AW, Voyles SV, Armstrong EJ, Fuller EN, Rutledge JC. Angiogenesis and oxidative stress: common mechanisms linking psoriasis with atherosclerosis. J Dermatol Sci. 2011;63:1-9.

25. Gelfand JM, et al. Risk of myocardial infarction in patients with psoriasis. JAMA. 2006;296:1735.

26. Henseler T, Christophers E. Psoriasis of early and late onset: characterization of two types of psoriasis vulgaris. J Am Acad Dermatol. 1985;13:450-6.

27. Ogdie $A$, et al. Prevalence and treatment patterns of psoriatic arthritis in the UK. Rheumatology. 2013;52:568-75.

28. Puig L. Cardiometabolic comorbidities in psoriasis and psoriatic arthritis. Int J Mol Sci. 2017;19:58.

29. Husted JA, et al. Cardiovascular and other comorbidities in patients with psoriatic arthritis: a comparison with patients with psoriasis. Arthritis Care Res. 2011;63:1729-35.

30. Greenland P, et al. ACCF/AHA guideline for assessment of cardiovascular risk in asymptomatic adults: a report of the american college of cardiology foundation/american heart association task force on practice guidelines. Circulation. 2010;122(2010):e50.

31. Kaiafa G, et al. Is anemia a new cardiovascular risk factor? Int I Cardiol. 2015; 186:117-24.

32. Ali N, Srey R, Pavlakis S. Hemoglobinopathies and stroke: strategies for prevention and treatment. Curr Treat Options Cardiovasc Med. 2012;14: 227-36.

33. Roubille C, et al. The effects of tumour necrosis factor inhibitors, methotrexate, non-steroidal anti-inflammatory drugs and corticosteroids on cardiovascular events in rheumatoid arthritis, psoriasis and psoriatic arthritis: a systematic review and meta-analysis. Ann Rheum Dis. 2015;74(3):480. https://doi.org/10.1136/annrheumdis-2014-206624

34. Caiazzo G, et al. Psoriasis, cardiovascular events, and biologics: lights and shadows. Front Immunol. 2018;9:1668.

35. Choi HK, Hernán MA, Seeger JD, Robins JM, Wolfe F. Methotrexate and mortality in patients with rheumatoid arthritis: a prospective study. Lancet. 2002:359:1173-7.

36. Chen Y-J, et al. Association between systemic antipsoriatic drugs and cardiovascular risk in patients with psoriasis with or without psoriatic arthritis: a nationwide cohort study. Arthritis Rheum. 2012;64:1879-87.

37. Ahlehoff $\mathrm{O}$, et al. Cardiovascular outcomes and systemic anti-inflammatory drugs in patients with severe psoriasis: 5-year follow-up of a Danish nationwide cohort. J Eur Acad Dermatol Venereol. 2015;29:1128-34.

38. Abuabara K, Lee $H$, Kimball AB. The effect of systemic psoriasis therapies on the incidence of myocardial infarction: a cohort study. Br J Dermatol. 2011; 165:1066-73.

39. Tardif J-C, et al. Efficacy and safety of low-dose colchicine after myocardial infarction. N Engl J Med. 2019;381(26):2497. https://doi.org/10.1056/ NEJMoa1912388

\section{Publisher's Note}

Springer Nature remains neutral with regard to jurisdictional claims in published maps and institutional affiliations.

Ready to submit your research? Choose BMC and benefit from:

- fast, convenient online submission

- thorough peer review by experienced researchers in your field

- rapid publication on acceptance

- support for research data, including large and complex data types

- gold Open Access which fosters wider collaboration and increased citations

- maximum visibility for your research: over $100 \mathrm{M}$ website views per year

At $\mathrm{BMC}$, research is always in progress.

Learn more biomedcentral.com/submissions 